\title{
Effect of pre-contraction on $\beta$-adrenoceptor-mediated relaxation of rat urinary bladder
}

\author{
Martin Christian Michel · Carsten Sand
}

Received: 21 January 2009 / Accepted: 24 April 2009 / Published online: 16 May 2009

(C) The Author(s) 2009. This article is published with open access at Springerlink.com

\begin{abstract}
Purpose The human physiological bladder contraction is largely mediated by acetylcholine acting on muscarinic receptors, but in pathophysiological settings the relative role of non-cholinergic stimuli gains importance. $\beta$-Adrenoceptor agonists are currently in clinical development as treatments for the overactive bladder syndrome. Therefore, we have explored the ability of the $\beta$-adrenoceptor agonist isoprenaline to induce rat isolated bladder strip relaxation on pre-contraction with the muscarinic agonist carbachol as compared to bladder tone induced by several non-cholinergic stimuli.

Methods Bladder tone was induced by passive tension, receptor independently by $\mathrm{KCl}$, carbachol, bradykinin or serotonin. Concentration-response curves were generated for relaxation by isoprenaline, and a single concentration of the receptor-independent relaxant forskolin was also tested. Results The various contractile stimuli induced different degrees of bladder tone, but the ability of isoprenaline or forskolin to relax rat bladder was not correlated with the degree of tone. Isoprenaline was significantly less potent and effective in causing relaxation against carbacholinduced tone than against any other stimulus, whereas no such relationship was observed for forskolin.

Conclusions We conclude that $\beta$-adrenoceptor agonists can induce rat bladder relaxation against a wide range of
\end{abstract}

\section{C. Michel ( $\square)$}

Department of Pharmacology and Pharmacotherapy,

Academic Medical Centre, University of Amsterdam,

1105 AZ Amsterdam, The Netherlands

e-mail: m.c.michel@amc.nl

C. Sand

Department of Pharmacology,

University of Duisburg-Essen, Essen, Germany contractile stimuli and are more potent and/or effective against non-cholinergic stimuli than against muscarinic agonism. This profile appears desirable for agents intended for the treatment of overactive bladder.

Keywords Bladder - Relaxation - Muscarinic · $\beta$-Adrenoceptor

\section{Introduction}

The physiological voiding contractions of the human urinary bladder are primarily elicited by the parasympathetic pelvic nerves, which release acetylcholine to act on muscarinic receptors, mainly of the $M_{3}$ subtype [1]. This is different from the situation in non-primate species, where non-cholinergic agents contribute considerably to physiological voiding [2]. However, under pathophysiological conditions, the relative role of non-cholinergic stimuli in mediating bladder contraction increases also in humans [3-6]. This may at least partly explain why muscarinic receptor antagonists have only moderate efficacy relative to placebo in the treatment of the overactive bladder syndrome $(\mathrm{OAB})[7,8]$.

The storage function of the urinary bladder requires smooth muscle relaxation to accommodate increasing volumes of urine without major elevations of intravesical pressure [9]. $\beta$-Adrenoceptor stimulation provides an effective means to cause bladder smooth muscle relaxation, and, in most species including humans, this involves a strong $\beta_{3}$ adrenoceptor component [10]. Accordingly, $\beta_{3}$-adrenoceptorselective agonists are currently under clinical development for the treatment of $\mathrm{OAB}$, and clinical proof of concept data for their efficacy have recently been presented [11].

Treatments for $\mathrm{OAB}$ patients should inhibit non-voiding bladder contractions, but have only little effect against 
physiological voiding contractions of the bladder. The latter is largely mediated by muscarinic receptor stimulation, whereas the former involves non-cholinergic mechanisms to a major degree. One previous study in rat bladder has indicated that the $\beta$-adrenoceptor agonist isoprenaline is more potent against the receptor-independent contractile stimulus $\mathrm{KCl}$ than against the muscarinic receptor agonist carbachol [12]. While $\mathrm{KCl}$ is often used in experimental in vitro studies to induce smooth muscle contraction, it is unclear how this relates to pathophysiological non-cholinergic stimuli. Therefore, we compared the ability of the prototypical $\beta$-adrenoceptor agonist isoprenaline to induce carbachol-induced bladder contraction with that induced by a range of other stimuli.

\section{Materials and methods}

All experiments were performed according to the German legislation on experimental animals and with approval by the state animal welfare board. Male adult Wistar rats were obtained from the central animal breeding facility of the University of Duisburg-Essen. Relaxation experiments were performed as previously described [13] with minor modifications. Briefly, bladder strips (approximate diameter $1 \mathrm{~mm}$, length $20 \mathrm{~mm}$, weight $10 \mathrm{mg}$ ) were mounted under a resting tension of $10 \mathrm{mN}$ in $10 \mathrm{ml}$ organ baths containing Krebs-Henseleit buffer of the following composition (mM): $\mathrm{NaCl} 119, \mathrm{KCl} 4.7, \mathrm{MgSO}_{4} 1.2, \mathrm{Na}_{4}$ EDTA $0.027, \mathrm{CaCl}_{2} 2.5, \mathrm{KH}_{2} \mathrm{PO}_{4} 1.2, \mathrm{NaHCO}_{3} 25$, glucose 5.5 and HEPES $10 \mathrm{mM}$ at $37^{\circ} \mathrm{C}$ and continually gassed with $95 \% \mathrm{O}_{2}$ and $5 \% \mathrm{CO}_{2}$ to maintain a $\mathrm{pH}$ of 7.4. The bladder strips were allowed to equilibrate for $60 \mathrm{~min}$ during which the buffer was refreshed several times. Thereafter, the strips were stimulated three times with $50 \mathrm{mM} \mathrm{KCl}$ followed by $20 \mathrm{~min}$ of washout. At the end of the equilibration period, the buffer was replaced and passive tension was readjusted at $10 \mathrm{mN}$. Where indicated, $50 \mathrm{mM} \mathrm{KCl}, 1 \mu \mathrm{M}$ carbachol, $100 \mu \mathrm{M}$ serotonin or $3 \mu \mathrm{M}$ bradykinin were added; this induced an initial increase in the force of contraction, which partly abated to reach relatively stable plateau values. Those plateau values are depicted throughout the manuscript and were taken as baseline ( $0 \%$ relaxation) for all subsequent relaxation experiments. While it is theoretically possible that low and high agonist concentrations use different signaling pathways to elicit contraction, previous studies in the bladder do not support this notion [14]. Thereafter, cumulative concentration-response curves were generated for relaxation by isoprenaline; parallel strips not exposed to a $\beta$-adrenoceptor agonist served as time controls. To avoid desensitization, only a single concentrationresponse curve was generated for each bladder strip. At the end of each experiment, $10 \mu \mathrm{M}$ forskolin was added to each organ bath. Bradykinin, carbachol, forskolin, isoprenaline, serotonin and all other chemicals were from Sigma (Munich, Germany).

Concentration-response curves were analyzed by fitting sigmoidal curves to the pooled data. Relaxation responses to forskolin were expressed as percentage of the muscle tension prior to adding the $\beta$-adrenergic agonist. Relaxation responses to isoprenaline were expressed as the percentage of the forskolin response within the same muscle strip to control for strip-intrinsic differences in relaxation potential. Data are means \pm SEM of $n$ experiments. Statistical significance of group differences was found by one-way ANOVA followed by Dunnett's multiple comparison tests. A value of $P<0.05$ was considered to be significant. All curve fitting and statistical calculations were performed using the Prism program version 4.0 (Graphpad Software, San Diego, CA, USA).

\section{Results}

In the absence of bladder-contracting stimuli, passive tension was set to $10 \mathrm{mN}$. Bladder contraction was induced using $\mathrm{KCl}(50 \mathrm{mM})$, the muscarinic acetylcholine receptor agonist carbachol $(1 \mu \mathrm{M})$, serotonin $(100 \mu \mathrm{M})$ or bradyki$\operatorname{nin}(3 \mu \mathrm{M})$. The latter four initially induced a peak tension, which was followed by a decline to relatively stable plateau values over a period of approximately $20 \mathrm{~min}$. Thereafter, i.e., immediately prior to addition of isoprenaline, the force of contraction (including passive tension) was $40.2 \pm 1.7$, $24.0 \pm 1.1,11.5 \pm 0.3$ and $13.8 \pm 0.5 \mathrm{mN}$ for $\mathrm{KCl}$, carbachol, serotonin and bradykinin, respectively $(n=18-21)$. Under all five experimental conditions, bladder tone slightly decreased with time in the presence of the vehicle. The maximum decrease over a time course matching that for a concentration-response curve by isoprenaline was 13$23 \%$ with no statistically significant differences between groups (Table 1).

Isoprenaline concentration-dependently reduced bladder tension under all five experimental conditions (Fig. 1). Maximum relaxation and agonist potency were significantly lower when tested against carbachol than against all other conditions (Table 1). The combined effects of lower potency and efficacy mean that, e.g., an isoprenaline concentration of $100 \mathrm{nM}$ caused $75 \pm 3$ and $68 \pm 4 \%$ relaxation against passive tension or $\mathrm{KCl}$, respectively, but only $31 \pm 2 \%$ relaxation against carbachol. These differences are not explained by different degrees of tension prior to isoprenaline addition as neither maximum relaxation nor agonist potency was consistently related to the amount of tension (Fig. 2).

The relaxation by $10 \mu \mathrm{M}$ forskolin, as measured at the end of the concentration-response curves for isoprenaline, 
Table 1 Effects of pre-contraction on bladder relaxation by isoprenaline

\begin{tabular}{llll}
\hline & Time control & \multicolumn{2}{l}{ Isoprenaline } \\
\cline { 3 - 4 } & Maximum relaxation & $\mathrm{pEC}_{50}$ & $E_{\max }$ \\
\hline Passive tension & $21 \pm 4$ & $8.76 \pm 0.08^{*}$ & $84 \pm 2^{*}$ \\
KCl & $23 \pm 2$ & $8.00 \pm 0.06^{*}$ & $80 \pm 1^{*}$ \\
Carbachol & $13 \pm 2$ & $7.27 \pm 0.15$ & $57 \pm 2$ \\
Serotonin & $15 \pm 2$ & $8.54 \pm 0.05^{*}$ & $91 \pm 1^{*}$ \\
Bradykinin & $16 \pm 3$ & $8.66 \pm 0.10^{*}$ & $79 \pm 2^{*}$ \\
\hline
\end{tabular}

All $E_{\max }$ effects are expressed as the percentage of the effect of $10 \mu \mathrm{M}$ forskolin in a given preparation. " $E_{\max }$ " effects in time control experiments refer to time points matching those of the concentrationresponse curves for the agonists (see figures). Data are means \pm SEM of $6-8$ experiments

$* P<0.05$ versus carbachol-induced contraction in a one-way ANOVA followed by Dunnett's multiple comparison test

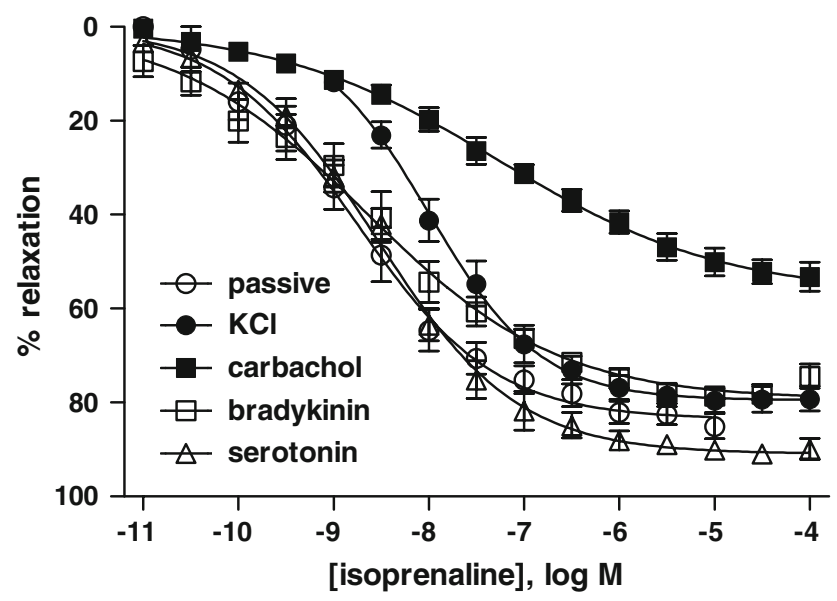

Fig. 1 Relaxation of rat urinary bladder strips by isoprenaline in relationship to pre-contraction stimulus. Data are means \pm SEM of 6-8 experiments. A quantitative analysis of the data is shown in Table 1

was $51 \pm 3,57 \pm 1,75 \pm 3,65 \pm 2$ and $70 \pm 2 \%$ for passive tone, $\mathrm{KCl}$, carbachol, serotonin and bradykinin, respectively $(n=18-24)$. Thus, forskolin-induced relaxation was stronger against carbachol, serotonin or bradykinin compared to that against passive tension $(P<0.01$ each versus passive tension in a one-way ANOVA). Again, this was not explained by different degrees of tension prior to adding the relaxing agents, as the extent of forskolininduced relaxation was not significantly related to the strength of tension (Fig. 2).

\section{Discussion}

Non-voiding contractions, i.e., detrusor overactivity, are believed to play a central role in the pathophysiology of the overactive bladder syndrome. While physiological voiding
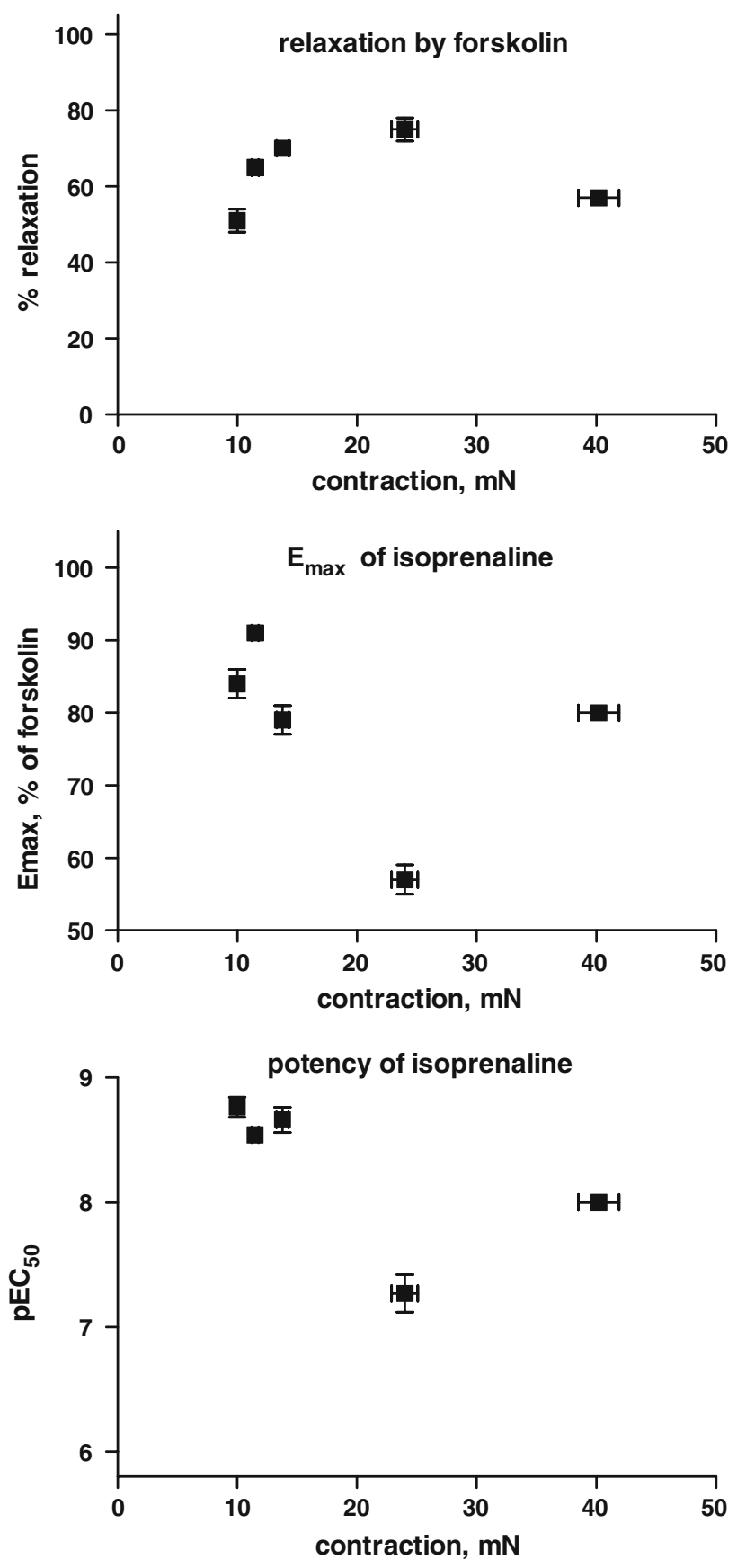

Fig. 2 Relationship between strength of pre-contraction on the relaxation responses to forskolin (top panel) and isoprenaline (middle panel for maximum effect and bottom panel for potency). Data are means \pm SEM of $6-8$ experiments

contractions of the human detrusor are largely mediated by muscarinic receptors, pathological non-voiding contractions involve non-cholinergic mechanisms to a much larger extent [3-6]. Treatments of the overactive bladder syndrome should suppress detrusor overactivity without impairing voiding, and $\beta$-adrenoceptor agonists are one of the future options for such treatments currently undergoing clinical investigation [11]. Against this background, the 
present study has systematically explored the role of pre-contracting stimulus on the ability of the prototypical $\beta$-adrenoceptor agonist isoprenaline to cause bladder smooth muscle relaxation.

The purinergic receptor agonist ATP may be one of the most important non-cholinergic pathophysiological stimuli to elicit bladder contraction [5]. However, purinergic stimuli typically elicit only phasic contractions, which do not allow testing relaxation against tension induced by them in our experimental design. Therefore, we chose to test a range of other non-cholinergic stimuli, which in total should provide a good picture of non-cholinergic contraction. As expected [9], these stimuli induced different degrees of bladder tone. However, neither the ability of the receptor-independent stimulus forskolin nor the potency or efficacy of isoprenaline to relax rat bladder strips was related to the extent of pre-contraction. These data demonstrate that factors distinct from the strength of pre-contraction contribute to differences in the ability of isoprenaline to cause bladder relaxation. Another methodological consideration is that not only the detrusor, but also the urothelium contains functional $\beta$-adrenoceptors [15] and that our experimental protocol has not systematically removed the urothelium. However, it can be assumed that any confounding effect of the urothelium was consistent across all experimental conditions.

To the best of our knowledge, only one previous study has compared relaxation against a muscarinic agonist with that against a non-cholinergic stimulus [12]. These authors found isoprenaline to be a weaker relaxant against carbachol than against the receptor-independent stimulus $\mathrm{KCl}$. This observation was confirmed in the present study, but it remains unclear how relevant $\mathrm{KCl}$ is as a comparative stimulus, because relaxation against $\mathrm{KCl}$ can differ from that against passive tension [16]. Therefore, we compared relaxation against carbachol with that against four other conditions and found that carbachol-induced tone was more difficult to relax by isoprenaline than that by any other stimulus. This "resistance" of carbachol-induced tone against relaxation did not apply to the receptor-independent relaxation by forskolin. As the autonomic regulation of smooth muscle tone in the bladder and airways has many similarities [17], it is interesting to note that studies on human airways has also reported that $\beta$-adrenoceptor agonists cause weaker relaxation against contraction induced by carbachol than against other stimuli [18, 19], thus indirectly supporting our findings. One possible mechanistic explanation of these findings is a specific interaction between $M_{2}$ muscarinic and $\beta$-adrenergic receptors as has been proposed in rats [20] and mice [21]. This may become important if the role of $\mathrm{M}_{2}$ receptors is enhanced under conditions of bladder overactivity [22]. The molecular signaling underlying relaxation responses by $\beta$-adrenoceptor agonists against various contractile stimuli has not been investigated in detail, but previous studies demonstrate that activation of $\mathrm{BK}_{\mathrm{Ca}}$ channels may play an important role, at least for the relaxation against $\mathrm{KCl}$-induced tone [16, 23].

In conclusion, our data show that a $\beta$-adrenoceptor agonist is more potent and more efficacious in relaxing rat bladder contraction induced by a range of non-cholinergic stimuli as compared to that induced by the muscarinic agonist carbachol. These findings raise the possibility that $\beta$ adrenoceptor agonists have a therapeutic window in which they can suppress detrusor overactivity without impairing physiological voiding. While the present rat study does not allow conclusions on the $\beta$-adrenoceptor subtype being targeted, the predominant role of $\beta_{3}$-adrenoceptors in the human bladder [10] suggests that a drug useful in patients should be $\beta_{3}$-selective. While this remains to be confirmed in urodynamic studies in patients, it is noteworthy that indeed the $\beta_{3}$-adrenoceptor agonist mirabegron was recently shown to effectively reduce voiding dysfunction in OAB patients without apparent interference with physiological voiding [11].

Acknowledgments This study was supported in part by grants from the Deutsche Forschungsgemeinschaft (Mi 294/7) and from Boehringer Ingelheim. The technical assistance of Ms. Dilek Tatli is gratefully acknowledged.

Conflict of interest statement In the field of bladder $\beta$-adrenoceptors, MCM has received research funds and/or consultancy honoraria from Astellas and Boehringer Ingelheim. CS does not report any conflict of interest.

Open Access This article is distributed under the terms of the Creative Commons Attribution Noncommercial License which permits any noncommercial use, distribution, and reproduction in any medium, provided the original author(s) and source are credited.

\section{References}

1. Hegde SS (2006) Muscarinic receptors in the bladder: from basic research to therapeutics. Br J Pharmacol 147:S80-S87. doi:10. 1038/sj.bjp.0706560

2. Sibley GN (1984) A comparison of spontaneous and nerve-mediated activity in bladder muscle from man, pig and rabbit. J Physiol 354:431-443

3. Fry CH, Wu C, Mundy AR (1999) Bladder instability and detrusor smooth muscle function. Exp Physiol 84:161-169

4. Yoshida M, Homma Y, Inadome A et al (2001) Age-related changes in cholinergic and purinergic neurotransmission in human isolated bladder smooth muscles. Exp Gerontol 36:99-109. doi:10.1016/S0531-5565(00)00175-3

5. Rapp DE, Lyon MB, Bales GT et al (2005) A role for the P2X receptor in urinary tract physiology and in the pathophysiology of urinary dysfunction. Eur Urol 48:303-308. doi:10.1016/j.eururo.2005.04.019

6. Yoshida M, Masunaga K, Satoji Y et al (2008) Basic and clinical aspects of non-neuronal acetylcholine: expression of non-neuronal acetylcholine in urothelium and its clinical significance. J Pharmacol Sci 106:193-198. doi:10.1254/jphs.FM0070115 
7. Chapple CR, Khullar V, Gabriel Z et al (2008) The effects of antimuscarinic treatments in overactive bladder: an update of a systematic review and meta-analysis. Eur Urol 54:543-562. doi:10.1016/j.eururo.2008.06.047

8. Novara G, Galfano A, Secco S et al (2008) A systematic review and meta-analysis of randomized controlled trials with antimuscarinic drugs for overactive bladder. Eur Urol 54:740-764. doi:10.1016/j.eururo.2008.06.080

9. Andersson K-E, Arner A (2004) Urinary bladder contraction and relaxation: physiology and pathophysiology. Physiol Rev 84:935986. doi:10.1152/physrev.00038.2003

10. Michel MC, Vrydag W (2006) $\alpha_{1^{-}}, \alpha_{2}$ - and $\beta$-adrenoceptors in the urinary bladder, urethra and prostate. Br J Pharmacol 147:S88S119. doi:10.1038/sj.bjp.0706619

11. Chapple CR, Yamaguchi O, Ridder A et al (2008) Clinical proof of concept study (Blossom) shows novel $\beta 3$ adrenoceptor agonist YM178 is effective and well tolerated in the treatment of symptoms of overactive bladder. Eur Urol Suppl 7:239

12. Longhurst PA, Levendusky M (1999) Pharmacological characterization of $\beta$-adrenoceptors mediating relaxation of the rat urinary bladder in vitro. Br J Pharmacol 127:1744-1750. doi:10.1038/ sj.bjp.0702709

13. Frazier EP, Schneider T, Michel MC (2006) Effects of gender, age and hypertension on $\beta$-adrenergic receptor function in rat urinary bladder. Naunyn Schmiedebergs Arch Pharmacol 373:300-309. doi:10.1007/s00210-006-0077-y

14. Frazier EP, Peters SLM, Braverman AS et al (2008) Signal transduction underlying control of urinary bladder smooth muscle tone by muscarinic receptors and $\beta$-adrenoceptors. Naunyn Schmiedebergs Arch Pharmacol 377:449-462. doi:10.1007/s00210-0070208-0

15. Otsuka A, Shinbo H, Matsumoto R et al (2008) Expression and functional role of $\beta$-adrenoceptors in the human urinary bladder.
Naunyn Schmiedebergs Arch Pharmacol 377:473-481. doi:10.1007/s00210-008-0274-y

16. Frazier EP, Mathy M-J, Peters SLM et al (2005) Does cyclic AMP mediate rat urinary bladder relaxation by isoproterenol? J Pharmacol Exp Ther 313:260-267. doi:10.1124/jpet.104.077768

17. Michel MC, Parra S (2008) Similarities and differences in the autonomic control of airway and urinary bladder smooth muscle. Naunyn Schmiedebergs Arch Pharmacol 378:217-224. doi:10.1007/ s00210-008-0316-5

18. Sarria B, Naline E, Zhang Y et al (2002) Muscarinic $\mathrm{M}_{2}$ receptors in acetylcholine-isoproterenol functional antagonism in human isolated bronchus. Am J Physiol 283:L1125-L1132

19. Naline E, Trifilieff A, Fairhurst RA et al (2007) Effect of indacaterol, a novel long-acting $\beta_{2}$-agonist, on isolated human bronchi. Eur Respir J 29:575-581. doi:10.1183/09031936.00032806

20. Hegde SS, Choppin A, Bonhaus D et al (1997) Functional role of $\mathrm{M}_{2}$ and $\mathrm{M}_{3}$ muscarinic receptors in the urinary bladder of rats in vitro and in vivo. Br J Pharmacol 120:1409-1417. doi:10.1038/ sj.bjp. 0701048

21. Ehlert FJ, Ahn S, Pak KJ et al (2007) Neuronally released acetylcholine acts on the $\mathrm{M}_{2}$ muscarinic receptor to oppose the relaxant effect of isoproterenol on cholinergic contractions in mouse urinary bladder. J Pharmacol Exp Ther 322:631-637. doi:10.1124/ jpet.107.121756

22. Braverman AS, Doumanian LR, Ruggieri MR Sr (2006) $M_{2}$ and $\mathrm{M}_{3}$ muscarinic receptor activation of urinary bladder contractile signal transduction. II. Denervated rat bladder. J Pharmacol Exp Ther 316:875-880. doi:10.1124/jpet.105.094961

23. Uchida $H$, Shishido K, Nomiya $M$ et al (2005) Involvement of cyclic AMP-dependent and -independent mechanisms in the relaxation of rat detrusor muscle via $\beta$-adrenoceptors. Eur J Pharmacol 518:195-202. doi:10.1016/j.ejphar.2005.06.029 